\title{
The importance of age-specific data in routine syndromic surveillance
}

\author{
Roger Morbey*, Alex J. Elliot and Gillian E. Smith
}

Public Health England, Birmingham, United Kingdom

\section{Objective}

To investigate whether aberration detection methods for syndromic surveillance would be more useful if data were stratified by age band.

\section{Introduction}

When monitoring public health incidents using syndromic surveillance systems, Public Health England (PHE) uses the age of the presenting patient as a key indicator to further assess the severity, impact of the incident, and to provide intelligence on the likely cause. However the age distribution of cases is usually not considered until after unusual activity has been identified in the allages population data. We assessed whether monitoring specific age groups contemporaneously could improve the timeliness, specificity and sensitivity of public health surveillance.

\section{Methods}

First, we examined a wide range of health indicators from the PHE syndromic surveillance systems to identify for further study those with the greatest seasonal variation in the age distribution of cases. Secondly, we examined the identified indicators to ascertain whether any age bands consistently lagged behind other age bands. Finally, we applied outbreak detection methods retrospectively to age specific data, identifying periods of increased activity that were only detected or detected earlier when age-specific surveillance was used.

\section{Results}

Seasonal increases in respiratory indicators occurred first in younger age groups, with increases in children under 5 providing early warning of subsequent increases occurring in older age groups. Also, we found age specific indicators improved the specificity of surveillance using indicators relating to respiratory and eye problems; identifying unusual activity that was less apparent in the all-ages population.

\section{Conclusions}

Routine surveillance of respiratory indicators in young children would have provided early warning of increases in older age groups, where the burden on health care usage, e.g. hospital admissions, is greatest. Furthermore this cross-correlation between ages occurred consistently even though the age distribution of the burden of respiratory cases varied between seasons. Age specific surveillance can improve sensitivity of outbreak detection although all-age surveillance remains more powerful when case numbers are low.

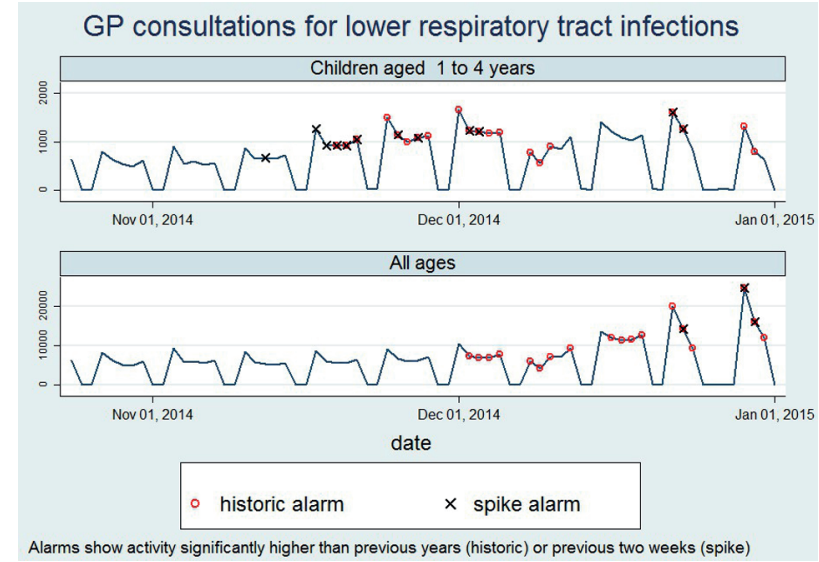

Keywords

Syndromic; Surveillance; Age

\section{Acknowledgments}

The authors are partly funded by the National Institute for Health Research (NIHR) Health Protection Research Unit in Emergency Preparedness and Response. The views expressed are those of the authors and not necessarily those of the National Health Service, the NIHR, the Department of Health or Public Health England.

\section{${ }^{*}$ Roger Morbey}

E-mail: roger.morbey@phe.gov.uk 